\title{
High-throughput quantitative analysis of pharmaceutical compounds in biological matrices
}

"The development of sub-2- $\mu m$ column, fused core column, LC-multiplexing and rapid fire TOF-MS approaches have really contributed to reducing the analysis time and therefore increasing the analysis throughput."

Keywords: biological matrices $\approx$ high-throughput analysis $\approx$ ionization at ambient temperature - LC $=$ MS $\approx$ ultra-fast chromatography

There has been a significant increase in the demand for new drug candidates exhibiting improved efficacy, selectivity and safety. Thus, the number of samples to be analyzed has considerably increased during the last 10 years. As a consequence, faster analytical methods are needed to evaluate as many of the new chemical entities as possible in order to select the lead candidates with the best metabolic and pharmacokinetic properties for clinical trials. After the biological samples have been cleaned up, compounds of interest are analyzed by LC-MS/MS, which has been used extensively for the quantitative determination of small molecules in biological matrices.

The introduction of atmospheric pressure ionization sources has alleviated the sensitivity, the selectivity and the robustness of LC-MS/MS assays [1]. Recent years have seen the introduction of a new generation of high-performance LC (HPLC) instruments that surpass the pressure limitation of conventional equipment (400 bar) and are capable of achieving liquid chromatographic separations at back pressures up to approximately 1000 bar. This technology has enabled the use of small particle size $(\leq 2 \mu \mathrm{m})$ stationary phase materials to obtain very high resolution separations [2]. One alternative stationary phase, so called fused-core particles, has been recently introduced and is marketed as $\mathrm{Halo}^{\mathrm{TM}}$ (MAC-MOD Analytical Inc. and Hichrom Ltd) or Ascentis Express ${ }^{\mathrm{TM}}$ (Sigma-Aldrich Co.). This material is a $2.7-\mu \mathrm{m}$ diameter particle consisting of a $0.5-\mu \mathrm{m}$ radius 'shell' of porous stationary phase surrounding a $1.7-\mu \mathrm{m}$ nonporous core. These particles exhibit reduced diffusional mass transfer compared with wholly porous particles and enable the use of higher mobile phase flows without concurrent loss of efficiency. In addition, with this type of stationary phase, similar peak capacities to those of several sub-2- $\mu \mathrm{m}$ porous particulate materials were observed in fast gradient analyses while maintaining lower back pressures $[3,4]$. While the run time in this type of LC-MS/MS is reduced to a minute in some cases, it takes only a few seconds for the mass spectrometer to record the chromatographic peak of interest [3]. This means that most of the time the mass spectrometer is idle, waiting for the next sample to come. As a consequence, the instrument time is not efficiently used and this reduces the sample throughput. In order to shorten this period of time where the MS detector is not acquiring data in conventional LC-MS/MS assays, multiplexing or parallel LC-MS/MS has been proposed. The concept of multiplexing originated from taking advantage of the difference between the chromatography run time and the MS data acquisition time. In multiplexing setups, multiple LC systems or columns are connected in parallel to a single mass spectrometer. Samples are introduced to the LC systems in a staggered fashion so that the analyte reaches the mass spectrometer from each LC system in serial order without overlapping. Multiple samples can be processed within the same time period required to analyze one sample with a single LC system. Another approach, so-called rapid fire, was also proposed recently. Contrary to multiplexing, rapid fire only utilizes one pump, with the chromatography column and the autosampler embedded in the system. All injection and switching valves as well as the chromatographic column are incorporated into the autosampler arm and, in conjunction with the

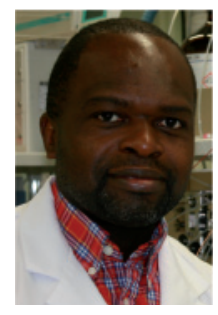

Olivier Heudi

Novartis Pharma AG, DMPK/ Bioanalytics, Forum I Novartis Campus, CH-4056 Basel,

Switzerland

Tel: +4I 79535961 I

Fax: +41 616968584

olivier.heudi@novartis.com 
ultra-fast robotics, drastically reduce cycle time. Thus, samples can be analyzed within $10 \mathrm{~s}$ [5]. So far, rapid fire technology in combination with TOF-MS has been successfully applied to a wide range of absorption, distribution, metabolism and excretion assays, including drug-drug interaction, metabolic stability, Caco-2, PAMPA and others. All methods using an autosampler are likely to suffer from carryover [5], which can then bias the absolute quantitative analysis results for small molecules in various biological matrices. Thus, a high-throughput method that does not use autosampler will offer an obvious advantage.

Recently, a large family of ambient ionization methods has been reviewed [6], namely desorption electrospray ionization (DESI), which depends on a solvent spray, and direct analysis in real time (DART), which utilizes plasma to create gas phase ions. These two techniques have been used for quantitative analysis work. With these two ambient methods no chromatographic or injection steps are required. The ionization of untreated samples occurs in the open environment. Thus, there is no requirement for sample preparation and, as a result, analysis is rapid with the time scale being governed by the time needed to present the sample to the mass spectrometer. High throughput (total analysis speed typically less than $5 \mathrm{~s}$ ) is a direct consequence of these features. DESI and DART display excellent sensitivity with absolute detection limits for pure compounds often in the sub-nanogram range [7]. However, accuracy and precision in complex matrices is controlled by the nature of the internal standard. The performance of these methods can be enhanced by the use of stableisotope-labeled compounds as internal standards. MALDI is one of the most commonly used soft ionization methods in MS. Moreover, the recent development of MALDI imaging and its popularity have generated renewed interest in MALDI techniques. MALDI-MS is a versatile tool for the detection of large proteins as well as many small molecules (e.g., in vitro drugs or metabolites). Recently, the FlashQuant ${ }^{\mathrm{TM}}$, a MALDI triple quadrupole, was lunched by AB/MDS Sciex. The first great benefit of this combination results from the intrinsic selectivity of selected reaction monitoring (SRM). This approach would considerably reduce the interfering MALDI matrix background. The second benefit is the high speed capability of the system, which would then allow high-throughput quantitative analysis. In most of the quantitative works with this approach, it was essential to use an internal standard to obtain good accuracy and precision data [8]. In addition, the choice of the ionization matrices is critical for the analysis as the MALDI methods suffer from huge matrix effect, which makes the assay not sensitive enough. Finally, quantitative analysis with the FlashQuant ${ }^{\mathrm{TM}}$ was not that successful and this is most probably the reason why $A B$ Sciex decided to discontinue the production of FlashQuant a couple of years ago.

Laser diode thermal desorption (LDTD) is a relatively new sample introduction source that does not require a HPLC step prior to detection via MS/MS. Thermal desorption of the analyte is initiated by the use of an infrared laser. This generates neutral molecules in the gas phase from samples that have been adsorbed onto a metallic surface; in the case of LDTD this is a specially designed stainless steel 96- or 384well plate. When combined with APCI these neutral gas-phase molecules can be ionized for subsequent detection by MS/MS. The direct nature of LDTD means that analysis times can range from 5 to $30 \mathrm{~s}$. LDTD has been mostly used to increase the throughput of in vitro assays, but recently some works have described the use of LDTD-APCI for the quantitative analysis of small molecules in biological matrices. The accuracy and precision data were similar to those observed with conventional LC-MS/MS assays $[9,10]$, and carryover was not observed when using LDTD-APCI.

The timeliness of result delivery concerning quantitative analysis of pharmaceutical compounds is predominantly dictated by the analysis of samples and data processing, and for this reason, breakthroughs in analysis technology have been of recent interest. Some notable examples in the area of chromatography, such as the development of a sub-2- $\mu$ m column and a fused core column, LC-multiplexing and rapid fire TOF-MS approaches, have really contributed to reducing analysis time and therefore increasing the analysis throughput. The development of new analytical techniques that do not use chromatography steps appears interesting and most of the quantitative works performed with these techniques are either semiquantitative or dedicated to absorption, distribution, metabolism and excretion or in vitro assays. Nevertheless, recent work has demonstrated their usefulness for quantitative bioanalysis. The sensitivity and selectivity of these assays still need to be improved as compared with classical LC-MS/MS methods. In addition, the complexity of the analyzed matrices and the lack of the time-resolved nature 
of conventional (LC-MS) analyses can impact the selective detection of isobaric species, even with high-resolution MS. The choice of one or other type of approach should be dictated by the ultimate purpose of the analysis, the type of samples (clinical or nonclinical) and the knowledge that bioanalysts have from the samples (metabolism or unstable metabolites). We should always keep in mind that the speed of the analysis could not go beyond the quality of the data generated.

\section{Financial \& competing interests disclosure}

The author has no relevant affiliations or financial involvement with any organization or entity with a financial interest in or financial conflict with the subject matter or materials discussed in the manuscript. This includes employment, consultancies, honoraria, stock ownership or options, expert testimony, grants or patents received or pending, or royalties.

No writing assistance was utilized in the production of this manuscript.

\section{Bibliography}

1 Jemal M. High-throughput quantitative bioanalysis by LC-MS/MS. Biomed. Chromatogr. 14, 422-429 (2000).

2 Yu K, Little D, Plumb R, Smith B. Highthroughput quantification for a drug mixture in rat plasma - a comparison of Ultra Performance ${ }^{\mathrm{TM}}$ liquid chromatography/ tandem mass spectrometry with highperformance liquid chromatography/tandem mass spectrometry. Rapid Comm. Mass Spectrom. 20, 544-552 (2006).

3 Badman ER, Beardsley RL, Liang Z, Bansal S. Accelerating high quality bioanalytical LC-MS/MS assays using fused-core columns. J. Chromatogr. B Analyt. Technol. Biomed. Life Sci. 878, 2307-2313 (2010).

4 Mallett DN, Ramirez-Molina C. The use of partially porous particle columns for the routine, generic analysis of biological samples for pharmacokinetic studies in drug discovery by reversed-phase ultra-high performance liquid chromatography-tandem mass spectrometry. J. Pharm. Biomed. Anal. 49, 100-107 (2009).

5 Brown A, Bickford S, Hatsis P, Amin J, Bell L, Harriman S. High-throughput analysis of in vitro cytochrome $\mathrm{p} 450$ inhibition samples using mass spectrometry coupled with an integrated liquid chromatography/autosampler system. Rapid Commun. Mass Spectrom. 24, 1207-1210 (2010).

6 Weston DJ. Ambient ionization mass spectrometry: current understanding of mechanistic theory; analytical performance and application areas. Analyst 135, 661-668 (2010).

7 Kauppila TJ, Wiseman JM, Ketola RA, Kotiaho T, Cooks RG, Kostiainen R. Desorption electrospray ionization mass spectrometry for the analysis of pharmaceuticals and metabolites. Rapid. Comm. Mass. Spectrom. 20, 387-392 (2006).
8 Wagner M, Varesio E, Hopfgartner G. Ultra-fast quantitation of saquinavir in human plasma by matrix-assisted laser desorption/ionization and selected reaction monitoring mode detection. J. Chromatogr. $B$ Analyt. Technol. Biomed. Life Sci. 872, 68-76 (2008).

9 Heudi O, Barteau S, Picard P, Tremblay P, Picard F, Kretz O. Laser diode thermal desorption-positive mode atmospheric pressure chemical ionization tandem mass spectrometry for the ultra-fast quantification of a pharmaceutical compound in human plasma. J. Pharm. Biomed. Anal. 54, 1088-1095 (2011).

10 Swales JG, Gallagher R, Peter RM. Determination of metformin in mouse, rat, dog and human plasma samples by laser diode thermal desorption/atmospheric pressure chemical ionization tandem mass spectrometry. J. Pharm. Biomed. Anal. 53, 740-744 (2010). 